\title{
Structural MRI Signatures in Genetic Presentations of the Frontotemporal Dementia/ Motor Neuron Disease Spectrum
}

\author{
Edoardo Gioele Spinelli, MD, Alma Ghirelli, MD, Silvia Basaia, PhD, Camilla Cividini, MSc, Nilo Riva, MD, PhD, \\ Elisa Canu, PhD, Veronica Castelnovo, MSc, Teuta Domi, PhD, Giuseppe Magnani, MD, \\ Francesca Caso, MD, PhD, Paola Caroppo, MD, PhD, Sara Prioni, MSc, Giacomina Rossi, PhD, \\ Lucio Tremolizzo, MD, Ildebrando Appollonio, MD, Vincenzo Silani, MD, Paola Carrera, BSc, \\ Massimo Filippi, MD, and Federica Agosta, MD, PhD
}

Neurology ${ }^{\circledR}$ 2021;97:e1594-e1607. doi:10.1212/WNL.0000000000012702

\section{Abstract}

\section{Background and Objectives}

To assess cortical, subcortical, and cerebellar gray matter (GM) atrophy using MRI in patients with disorders of the frontotemporal lobar degeneration (FTLD) spectrum with known genetic mutations.

\section{Methods}

Sixty-six patients carrying FTLD-related mutations were enrolled, including 44 with pure motor neuron disease (MND) and 22 with frontotemporal dementia (FTD). Sixty-one patients with sporadic FTLD (sFTLD) matched for age, sex, and disease severity with genetic FTLD (gFTLD) were also included, as well as 52 healthy controls. A whole-brain voxel-based morphometry (VBM) analysis was performed. GM volumes of subcortical and cerebellar structures were obtained.

\section{Results}

Compared with controls, GM atrophy on VBM was greater and more diffuse in genetic FTD, followed by sporadic FTD and genetic MND cases, whereas patients with sporadic MND (sMND) showed focal motor cortical atrophy. Patients carrying C9orf72 and GRN mutations showed the most widespread cortical volume loss, in contrast with GM sparing in SOD1 and TARDBP. Globally, patients with gFTLD showed greater atrophy of parietal cortices and thalami compared with sFTLD. In volumetric analysis, patients with gFTLD showed volume loss compared with sFTLD in the caudate nuclei and thalami, in particular comparing C9-MND with sMND cases. In the cerebellum, patients with gFTLD showed greater atrophy of the right lobule VIIb than sFTLD. Thalamic volumes of patients with gFTLD with a C9orf72 mutation showed an inverse correlation with Frontal Behavioral Inventory scores.

\section{Discussion}

Measures of deep GM and cerebellar structural involvement may be useful markers of gFTLD, particularly C9orf72-related disorders, regardless of the clinical presentation within the FTLD spectrum.

\author{
Correspondence \\ Dr. Agosta \\ agosta.federica@hsr.it
}




\section{Glossary}

ALS = amyotrophic lateral sclerosis; ALSFRS-r = ALS Functional Rating Scale-revised; ANOVA = analysis of variance; bvFTD = behavioral variant of frontotemporal dementia; CDR-FTLD = Clinical Dementia Rating-FTLD; FOV = field of view; FTD = frontotemporal dementia; FTLD = frontotemporal lobar degeneration; gFTLD = genetic frontotemporal lobar degeneration; $\mathbf{G M}=$ gray matter; $\mathbf{M N D}=$ motor neuron disease; $\mathbf{n f v P P A}=$ nonfluent variant primary progressive aphasia; PLS = primary lateral sclerosis; PMA = progressive muscular atrophy; PPA = primary progressive aphasia; $\mathbf{S F T D}=$ sporadic frontotemporal dementia; sFTLD = sporadic frontotemporal lobar degeneration; sMND = sporadic motor neuron disease; TE = echo time; $\mathbf{T R}=$ repetition time; $\mathbf{V B M}=$ voxel-based morphometry .

The successful identification of new therapies for frontotemporal lobar degeneration (FTLD) critically depends on the ability to identify useful markers mirroring specific biological processes within this heterogeneous spectrum of clinical syndromes. Cases due to genetic mutations represent up to $30 \%$ of FTLD presentations, ${ }^{1}$ and provide an ideal model for studying these processes, as the underlying pathology can be inferred (even in the absence of postmortem confirmation), facilitating the design of therapeutic trials targeting specific molecular mechanisms. Neuroimaging has been demonstrated to provide in vivo noninvasive measures of neurodegeneration in FTLD phenotypes. ${ }^{2}$ Distinctive patterns of atrophy on structural MRI contribute to establishing the correct diagnosis of behavioral variant of frontotemporal dementia (bvFTD) and primary progressive aphasia (PPA), ${ }^{3,4}$ help distinguish patients with FTLD from those with different pathologies, ${ }^{5}$ and provide hints about the underlying genetic and pathologic substrate of each patient. ${ }^{6}$ Only recently, the role of pathologic burden within gray matter (GM) structures other than the brain cortex has been investigated, suggesting a distinctive degeneration of deep GM and cerebellar structures in genetic FTLD (gFTLD) presentations, ${ }^{7-9}$ including motor neuron disease (MND). ${ }^{10}$ However, most previous studies were mainly focused on pure cognitive phenotypes, ${ }^{7,8,11}$ and an extensive characterization of subcortical and cerebellar damage across genetic forms of the frontotemporal dementia (FTD)/MND spectrum (including C9orf72-related disorders) is still in progress.

The aim of this study was to explore the neuroanatomical structural correlates of genetic heterogeneity in a cohort of patients affected by the wide spectrum of FTLD disorders, including MND. More specifically, we assessed with a systematic approach the patterns of atrophy of cortical, subcortical, and cerebellar structures using up-to-date MRI volumetric techniques to identify neuroimaging measures associated with specific genetic alterations.

\section{Methods}

\section{Participants}

A total of 658 patients with a suspected diagnosis of FTLDrelated disorders were prospectively enrolled in 4 referral clinics in Lombardy, Italy, and referred to San Raffaele Hospital in Milan between October 2007 and July 2019 to undergo MRI on a $3 \mathrm{~T}$ scanner, as part of diagnostic workup. Of these, 362 patients gave consent to be screened for known pathogenic mutations and evaluated for inclusion in the present multicenter, case-control study. Patients who underwent genetic screening had received a clinical diagnosis of FTD $(n=110)$ according to either bvFTD ${ }^{3}$ or $\mathrm{PPA}^{4}$ clinical criteria, or MND variants $(\mathrm{n}=252)$, including amyotrophic lateral sclerosis (ALS), ${ }^{12}$ progressive muscular atrophy (PMA), ${ }^{13}$ and primary lateral sclerosis (PLS) ${ }^{14}$ Sixty-six mutation carriers were identified, of which 22 presented with a clinical variant of FTD (i.e., bvFTD, $\mathrm{n}=12$; bvFTD-ALS, $\mathrm{n}=5$; nonfluent variant primary progressive aphasia [nfvPPA], $n=3$; $s v P P A, n=1$; and right-predominant variant of svPPA, $n=1$ ) and 44 with a pure MND phenotype (i.e., ALS, $n=35$; PMA, $n=6$; and PLS, $n=$ 3). Patients fulfilling both bvFTD ${ }^{3}$ and ALS criteria ${ }^{12}$ were considered as FTD for the subsequent MRI analyses, considering that greater atrophy was expected compared with pure MND. Figure 1A outlines the screening process of gFTLD, whereas Table 1 and eTables 1 and 4 (available from Dryad doi. org/10.5061/dryad.1vhhmgqsz) summarize the demographic and clinical features of included patients. Among patients who proved negative for known pathogenic mutations (i.e., sporadic FTLD [sFTLD]), we selected 61 patients to be matched with gFTLD cases for age, sex, and MRI scanner type, with comparable clinical diagnoses and disease severity assessed by Clinical Dementia Rating-FTLD (CDR-FTLD) scale ${ }^{15}$ for FTD and ALS Functional Rating Scale-revised (ALSFRS-r) ${ }^{16}$ for MND. Therefore, 16 sporadic FTD (sFTD, including bvFTD, $\mathrm{n}=12$; nfvPPA, $\mathrm{n}=2$; and svPPA, $\mathrm{n}=2$ ) and 45 sporadic pure MND (sMND) cases (i.e., ALS, $\mathrm{n}=37$; PMA, $\mathrm{n}$ $=5$; PLS, $\mathrm{n}=3$ ) were included (Table 1$)$. All patients underwent neurologic examination, multidomain cognitive testing, and brain MRI at study entry. Fifteen patients with sFTD (i.e., all but one with svPPA) and 10 patients with gFTD also underwent lumbar puncture to exclude CSF biomarker profile suggestive of Alzheimer disease pathology as part of their diagnostic workup (Table 1). No patient showed a p-tau/A $\beta_{42}$ ratio $>0.13$, considered as pathologic. ${ }^{17}$

Fifty-two healthy controls comparable for age, sex, and MRI scanner type with patient groups were recruited by word of mouth among participants unrelated to the patient population. Controls were included if the following criteria were satisfied: normal neurologic assessment, Mini-Mental State Examination score $\geq 28$, and no family history of neurodegenerative diseases. 
A

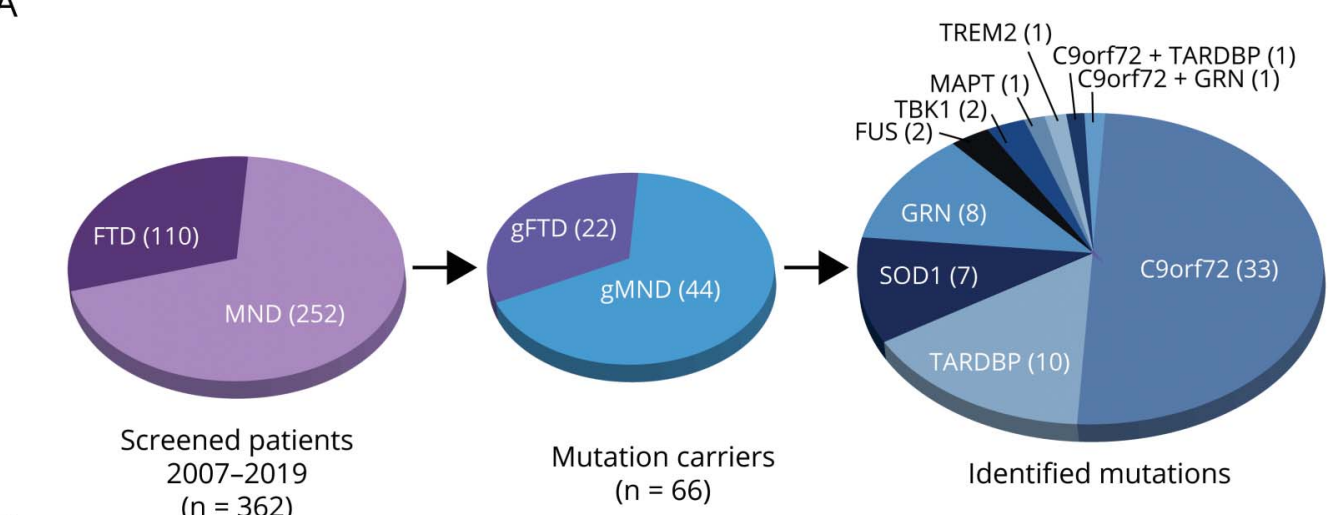

B

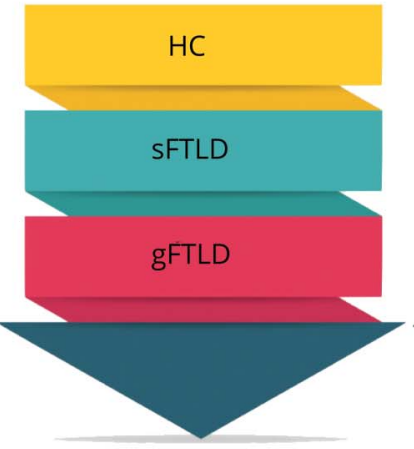

First level

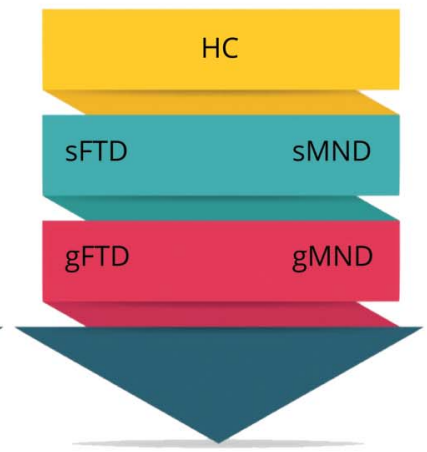

Second level

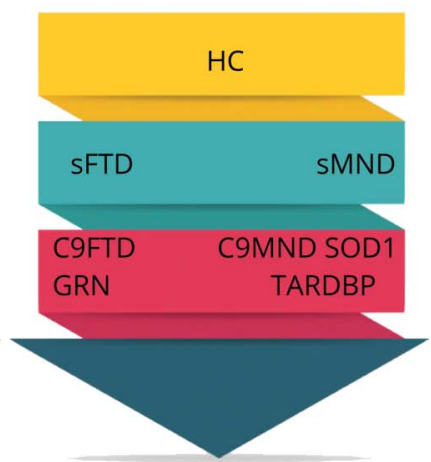

Third level

(A) A total of 362 patients with disorders of the frontotemporal lobar degeneration spectrum referred between 2007 and 2019 were screened for known pathogenic mutations. Sixty-six mutation carriers were identified. Detected mutations are reported in the last pie chart. (B) Diagram showing the hierarchical organization of the 3-level statistical analysis. FTD = frontotemporal dementia; gFTD = genetic frontotemporal dementia; gFTLD = genetic frontotemporal lobar degeneration; gMND = genetic motor neuron disease; $\mathrm{HC}=$ healthy control; $\mathrm{MND}=$ motor neuron disease; sFTD = sporadic frontotemporal dementia; sFTLD = sporadic frontotemporal lobar degeneration; SMND = sporadic motor neuron disease.

Exclusion criteria for all participants were medical illnesses or substance abuse that could interfere with cognitive functioning; any (other) major systemic, psychiatric, or neurologic illnesses; and other causes of focal or diffuse brain damage, including lacunae and extensive cerebrovascular disorders at routine MRI.

\section{Standard Protocol Approvals, Registrations, and Patient Consents}

The local ethical standards committee on human experimentation approved the study protocol and all participants provided written informed consent.

\section{Genetic Analysis}

Blood samples were collected from all patients and genomic DNA was obtained and processed in each of the recruiting centers. The presence of GGGGCC hexanucleotide expansion in the first intron of the C9orf72 gene was assessed using fluorescent amplicon-length analysis and a repeat-primed PCR assay. A cutoff of $\geq 30$ repeats combined with a typical sawtooth pattern was considered pathologic. In addition, GRN, MAPT, TARDBP, SOD1, FUS, TBK1, TREM2, OPTN, and $V C P$ genes were analyzed by next-generation sequencing and their mutations were confirmed by standard Sanger sequencing. All patients with MND were systematically tested for C9orf72, TARDBP, and SOD1 mutations, and additional testing of FUS and TBK1 was performed in the presence of positive family history of $\mathrm{MND} /$ dementia. Similarly, all patients with FTD were tested for C9orf72, TARDBP, MAPT, and GRN mutations, with additional testing of FUS, TBK1, TREM2, OPTN, and VCP in the presence of positive family history.

\section{Clinical and Neuropsychological Evaluations}

Clinical evaluation was performed by experienced neurologists blinded to MRI results. For patients presenting with FTD variants, disease severity was assessed using the CDRFTLD. ${ }^{15}$ For patients with MND, site of disease onset, disease severity using the ALSFRS-r, ${ }^{16}$ and manual muscle testing of strength based on the Medical Research Council scale were recorded. The rate of disease progression was defined according to the following formula: [48 - ALSFRS-r score]/ time from symptom onset.

Neuropsychological assessment was performed by experienced neuropsychologists unaware of MRI results. A 
Table 1 Main Sociodemographic and Clinical Characteristics of Healthy Controls and Patients With Frontotemporal Lobar Degeneration (FTLD) Classified by Clinical Presentation and Genetic Status

\begin{tabular}{|c|c|c|c|c|c|c|}
\hline & $\mathrm{HC}$ & SMND & gMND & sFTD & gFTD & $\begin{array}{l}p \\
\text { Value }\end{array}$ \\
\hline Number & 52 & 45 & 44 & 16 & 22 & \\
\hline Diagnosis & - & $\begin{array}{l}37 \text { ALS, } 5 \text { PMA, } 3 \\
\text { PLS }\end{array}$ & 35 ALS, 6 PMA, 3 PLS & $\begin{array}{l}12 \text { bvFTD, } 2 \text { nfvPPA, } 2 \\
\text { svPPA }\end{array}$ & $\begin{array}{l}12 \text { bvFTD, } 5 \text { bvFTD/ALS, } 1 \\
\text { svPPA, } 3 \text { nfvPPA, } 1 \text { R-SD }\end{array}$ & - \\
\hline$M / F$ & $26 / 26$ & $23 / 22$ & $22 / 22$ & $9 / 7$ & $11 / 11$ & 0.99 \\
\hline $\begin{array}{l}\text { Genetic } \\
\text { mutation }\end{array}$ & - & - & $\begin{array}{l}22 \text { C9orf72, } 10 \text { TARDBP, } 7 \text { SOD1, } 2 \\
\text { TBK1, } 2 \text { FUS, } 1 \text { C9orf72 + TARDBP }\end{array}$ & - & $\begin{array}{l}11 \text { C9orf72, } 8 \text { GRN, } 1 \text { MAPT, } 1 \\
\text { TREM2, } 1 \text { C9orf72 + GRN }\end{array}$ & - \\
\hline Family history ( \pm ) & $0 / 52$ & $3 / 42$ & $18 / 26^{a, b, c}$ & $2 / 14$ & $13 / 9^{a, b, c}$ & $<0.001$ \\
\hline Education, y & $\begin{array}{l}12.75 \pm 3.70 \\
(5-20)\end{array}$ & $\begin{array}{l}12.07 \pm 4.10 \\
(5-24)\end{array}$ & $10.84 \pm 3.23(5-20)$ & $10.36 \pm 3.81(5-17)$ & $11.11 \pm 4.19(5-21)$ & 0.13 \\
\hline Age at MRI, y & $\begin{array}{l}59.2 \pm 6.6 \\
(44.7-72.7)\end{array}$ & $\begin{array}{l}58.03 \pm 9.7 \\
(36-71)\end{array}$ & $57.25 \pm 10.01(31-75)$ & $61.4 \pm 5.7(46-71)$ & $60.26 \pm 4.78(49-67)$ & 0.34 \\
\hline $\begin{array}{l}\text { Disease } \\
\text { duration, mo }\end{array}$ & - & $\begin{array}{l}29.6 \pm 42.9 \\
(4-277)\end{array}$ & $25.70 \pm 26.57(4-112)$ & $30 \pm 10.4(22-48)$ & $29.35 \pm 30.35(9-119)$ & 0.96 \\
\hline CDR & - & - & - & $1.23 \pm 0.97(0-3)$ & $1.18 \pm 0.93(0-3)$ & 0.61 \\
\hline CDR-FTLD & - & - & - & $10.20 \pm 6.70(1-23)$ & $7.25 \pm 6.96(0.5-20)$ & 0.37 \\
\hline CDR-SB & - & - & - & $7.53 \pm 5.3(1-17)$ & $6.14 \pm 4.55(1-15)$ & 0.38 \\
\hline MMSE, \% ${ }^{e}$ & $\begin{array}{l}0.98 \pm 0.03 \\
(0.90-1)\end{array}$ & $\begin{array}{l}0.97 \pm 0.03 \\
(0.90-1)\end{array}$ & $0.96 \pm 0.05(0.71-1)$ & $\underset{a, b, d}{0.77} \pm 0.2(0.2-0.93)$ & $0.78 \pm 0.2(0.33-0.97)^{a, b, d}$ & 0.003 \\
\hline FBI total (0-72) & - & $1.95 \pm 1.83(0-6)$ & $2.82 \pm 3.13(0-13)$ & $28.00 \pm 11.6(15-45)^{b, d}$ & $17.33 \pm 13.09(0-35)^{b, d}$ & $<0.001$ \\
\hline ALSFRS-r (0-48) & - & $\begin{array}{l}37.36 \pm 6.2 \\
(23-47)\end{array}$ & $36.71 \pm 7.22(20-46)$ & - & $35.2 \pm 5.6(26-41)^{f}$ & 0.76 \\
\hline $\begin{array}{l}\text { MRC sum score } \\
(0-120)\end{array}$ & - & $\begin{array}{l}97.08 \pm 20.63 \\
(34-120)\end{array}$ & $95.25 \pm 20.96(41-120)$ & - & $90.4 \pm 12.58(78-110)^{f}$ & 0.77 \\
\hline $\begin{array}{l}\text { Disease } \\
\text { progression rate }\end{array}$ & - & $\begin{array}{l}0.64 \pm 0.6 \\
(0.07-2.4)\end{array}$ & $0.91 \pm 0.84(0.22-4)$ & - & $1.18 \pm 0.6(0.75-2.2)^{f}$ & 0.11 \\
\hline $\begin{array}{l}\text { Onset bulb/limb/ } \\
\text { bulb + limb }\end{array}$ & - & $8 / 36 / 1$ & $4 / 40 / 0$ & - & $2 / 3 / 0^{f}$ & - \\
\hline $\begin{array}{l}\text { CSF } \beta \text {-amyloid } 42, \\
\mathrm{pg} / \mathrm{mL}\end{array}$ & - & - & - & $\begin{array}{l}732.18 \pm 241.23 \\
(447-1,101)\end{array}$ & $785 \pm 325.9(452-1,470)$ & 0.68 \\
\hline $\begin{array}{l}\text { CSF total tau, pg/ } \\
\mathrm{mL}\end{array}$ & - & - & - & $\begin{array}{l}193.73 \pm 67.08 \\
(121-350)\end{array}$ & $286.3 \pm 177.24(72-751)$ & 0.13 \\
\hline $\begin{array}{l}\text { CSF } \\
\text { phosphorylated } \\
\text { tau, pg/mL }\end{array}$ & - & - & - & $31.49 \pm 12.51(15-57)$ & $38.7 \pm 16.62(20-64)$ & 0.28 \\
\hline
\end{tabular}

Abbreviations: ALS = amyotrophic lateral sclerosis; ALSFRS-r = Amyotrophic Lateral Sclerosis Functional Rating Scale, revised version; bvFTD = behavioral variant frontotemporal dementia; $\mathrm{CDR}=$ Clinical Dementia Rating scale; CDR-SB = Clinical Dementia Rating scale, sum of boxes; FBI = Frontal Behavioral Inventory; gFTD = genetic frontotemporal dementia; gMND = genetic motor neuron disease; HC = healthy control; MMSE = Mini-Mental State Examination; MND = motor neuron disease; MRC = Medical Research Council; nfvPPA = nonfluent variant primary progressive aphasia; PLS = primary lateral sclerosis; PMA = progressive muscular atrophy; R-SD = right-sided semantic dementia; sFTD = sporadic frontotemporal dementia; sMND = sporadic motor neuron disease; SVPPA = semantic variant primary progressive aphasia.

Values are reported as mean \pm SD (range). $p$ Values refer to analysis of variance models, Bonferroni-corrected for multiple comparisons.

a Statistically significant difference vs HC.

b Statistically significant difference vs SMND.

c Statistically significant difference vs sFTD.

d Statistically significant difference vs gMND.

e Ratio between correct and administered items, considering patient's motor disability.

${ }^{f}$ bvFTD/ALS cases.

comprehensive multidomain cognitive and behavioral battery was administered, as previously described. ${ }^{18}$ Based on available cognitive measures, a diagnosis according to the revised
Strong criteria for cognitive/behavioral impairment in MND $^{19}$ could be made for 30 patients with sMND and 29 patients with gMND. 
Figure 2 Patterns of Gray Matter (GM) Atrophy in Patients With Genetic and Sporadic Frontotemporal Lobar Degeneration (FTLD)

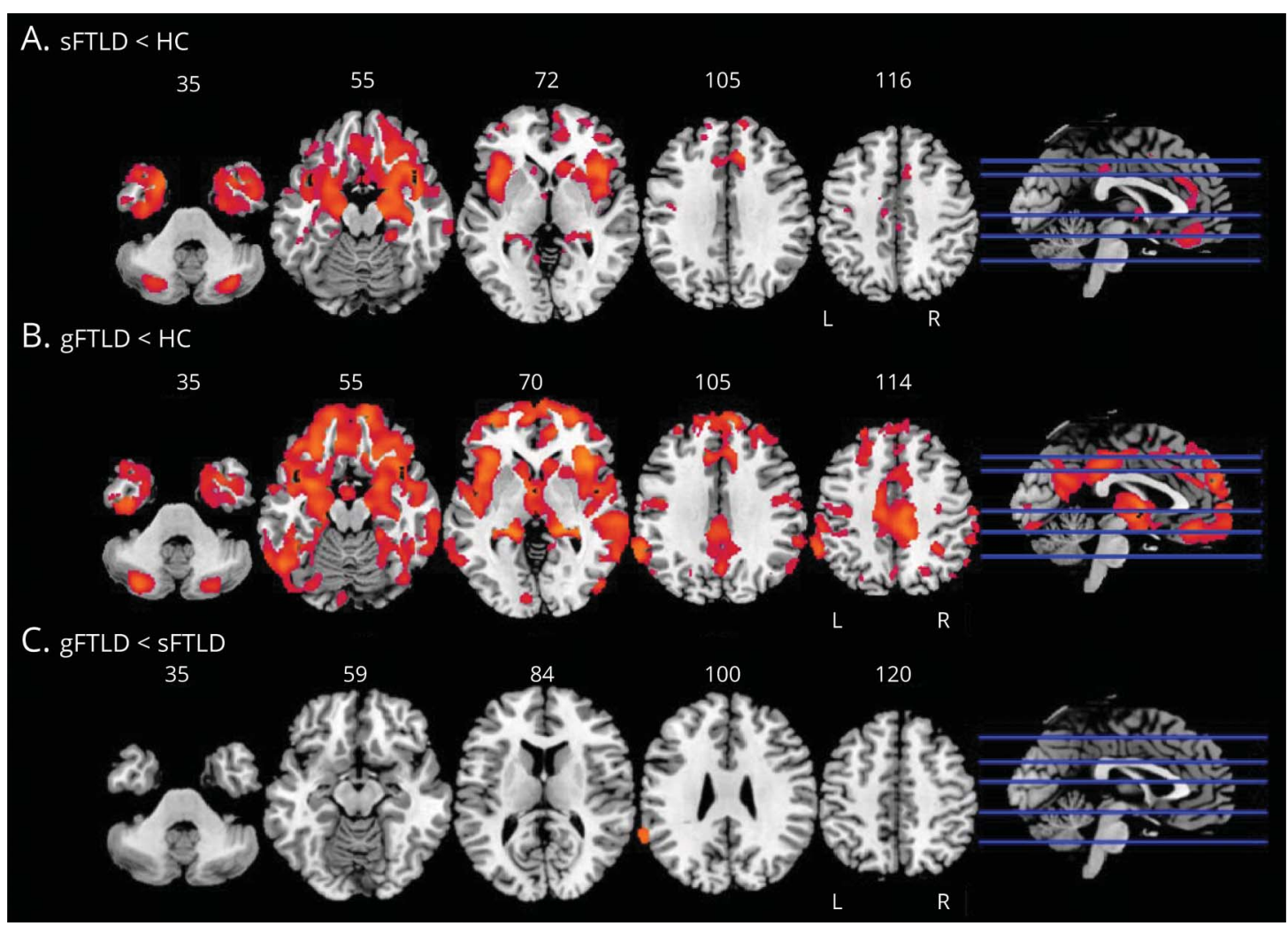

Results of voxel-based morphometry analysis showing regions of significant GM atrophy in sporadic FTLD (SFTLD) and genetic FTLD (gFTLD) when compared with healthy controls ( $\mathrm{HC}$ ) (A and B) and between each other (C). Significant clusters are overlaid on sections of the Montreal Neurologic Institute standard brain. Analyses were corrected for age, sex, and total intracranial volume. Statistical threshold for significance was $p<0.05$, family-wise error-corrected for multiple comparisons.

\section{MRI Acquisition}

All patients and healthy controls underwent brain MRI on a 3T scanner (Philips Medical Systems) at San Raffaele Hospital between 2007 and 2019. The original scanner (for brevity, scanner 1) was substituted with an upgraded model from the same manufacturer in 2016 (scanner 2).

Using scanner 1, a 3D T1-weighted fast field echo sequence was acquired (repetition time [TR] $25 \mathrm{~ms}$, echo time [TE] $4.6 \mathrm{~ms}$, flip angle $30^{\circ}, 220$ axial slices with voxel size $0.89 \times 0.89 \times$ $0.8 \mathrm{~mm}$, matrix size $256 \times 256$, field of view [FOV] $230 \times$ $182 \mathrm{~mm}^{2}$ ). Using scanner 2, a 3D T1-weighted turbo field echo with comparable resolution was acquired (TR $7 \mathrm{~ms}$, TE $3.2 \mathrm{~ms}$, inversion time $1,000 \mathrm{~ms}, 204$ sagittal slices with voxel size $1 \times 1$ $\times 1 \mathrm{~mm}$, matrix $256 \times 256$, FOV $256 \times 256 \mathrm{~mm}^{2}$ ).

\section{MRI Analysis}

\section{Voxel-Based Morphometry}

Voxel-based morphometry (VBM) was performed using SPM12 (fil.ion.ucl.ac.uk/spm/) and diffeomorphic anatomical registration exponentiated lie algebra (DARTEL) registration method ${ }^{20}$ to investigate GM volume alterations at a whole-brain level. Details of the VBM pipeline have been described previously. ${ }^{21}$

\section{Volumetric Analysis}

Volumes of the deep GM structures (i.e., bilateral caudate, globus pallidus, putamen, and thalamus), hippocampus, and amygdala were obtained using the FMRIB's Integrated Registration and Segmentation Tool (FIRST) in FSL (fmrib.ox.ac.uk) fsl/first/index.html). Local GM volumes of the cerebellar lobules and vermis were calculated automatically using an atlas propagation and label fusion strategy based on the SUIT atlas. ${ }^{22,23} \mathrm{GM}$ volumes were multiplied by the normalization factor derived from SIENAx (fmrib.ox.ac.uk/fsl/sienax/index.html) to correct for subject head size.

\section{Statistical Analysis}

Normal distribution assumption was checked by means of Q-Q plot and Shapiro-Wilk and Kolmogorov-Smirnov tests. Clinical, neuropsychological, and MRI volumetric measures were compared between groups using age-, sex-, and MRI scanner-adjusted analysis of variance (ANOVA) models, followed by post hoc pairwise comparisons, Bonferroni-corrected for multiple comparisons. The threshold of significance was set at $p<0.05$. SPSS Statistics 22.0 software was used.

VBM group comparisons were tested using ANOVA models in SPM12, adjusting for total intracranial volume, 
Figure 3 Patterns of Gray Matter (GM) Atrophy in Patients With Frontotemporal Lobar Degeneration (FTLD) According to Genetic Status and Clinical Presentation

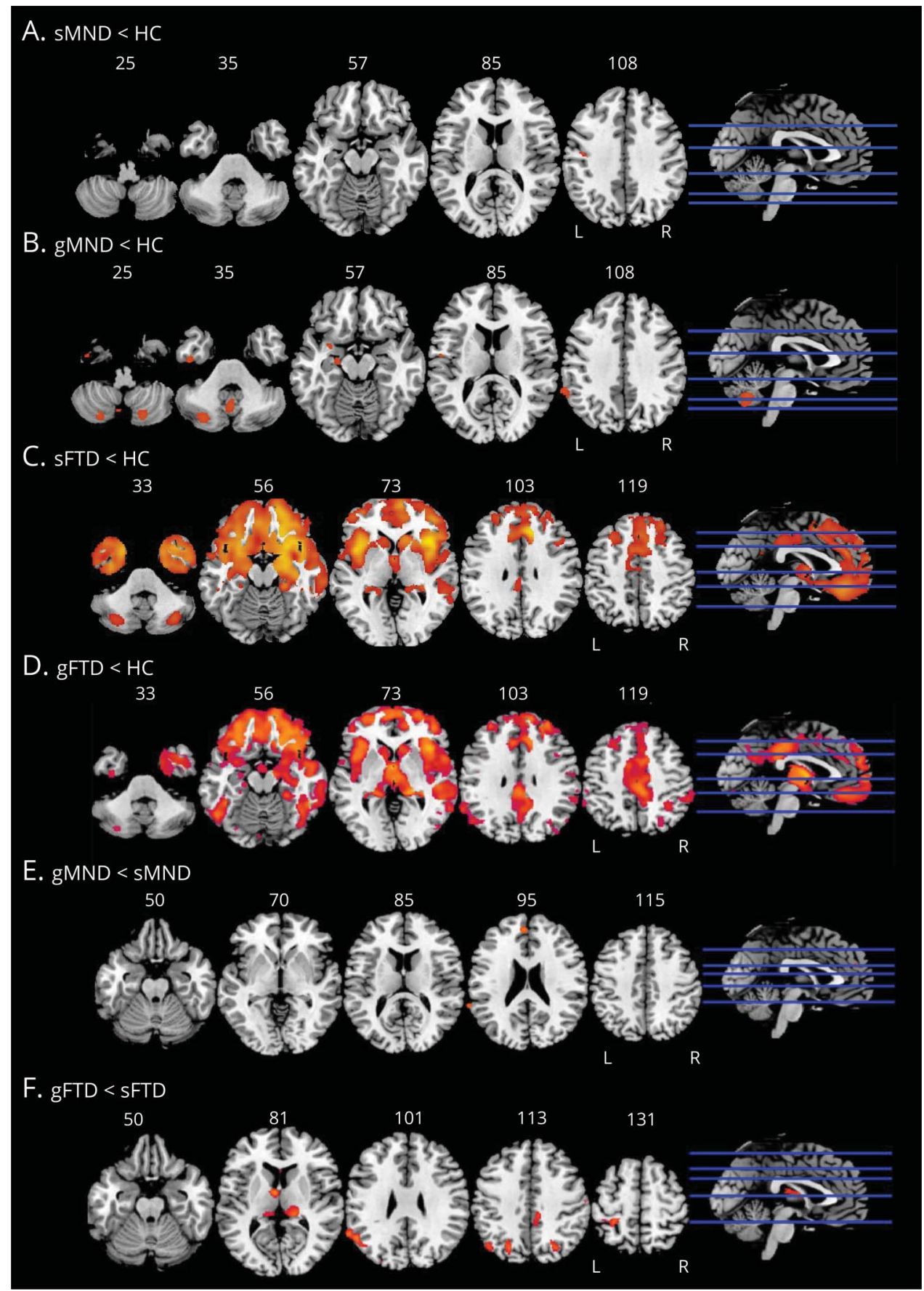

Results of voxel-based morphometry analysis showing regions of significant GM atrophy in sporadic and genetic frontotemporal dementia/motor neuron disease when compared with healthy controls (HCS) (A-D) and between each other $(E, F)$. Significant clusters are overlaid on sections of the Montreal Neurologic Institute standard brain. Analyses were corrected for age, sex, and total intracranial volume. Statistical threshold for significance was $p<0.05$, family-wise error-corrected for multiple comparisons. gFTD = genetic frontotemporal dementia; gMND = genetic motor neuron disease; SFTD $=$ sporadic frontotemporal dementia; sMND = sporadic motor neuron disease.

age, sex, and MRI scanner type. Results were assessed at $p<0.05$, family-wise error-corrected for multiple comparisons.

Correlations between clinical, cognitive, and MRI volumetric features of patients with FTLD (i.e., gFTLD and sFTLD, separately) were tested by means of partial correlation analyses adjusted for age, sex, and education. Subsequently, the same analysis was performed subdividing the gFTLD group according to the C9orf72 status. The threshold of statistical significance was set at $p<0.05$, Bonferronicorrected for multiple comparisons. SPSS Statistics 22.0 software was used.

\section{Data Availability}

The dataset used and analyzed during the current study is available from the corresponding author on reasonable request. 
Figure 4 Patterns of Gray Matter (GM) Atrophy in Patients With Frontotemporal Lobar Degeneration (FTLD) According to Genetic Mutation

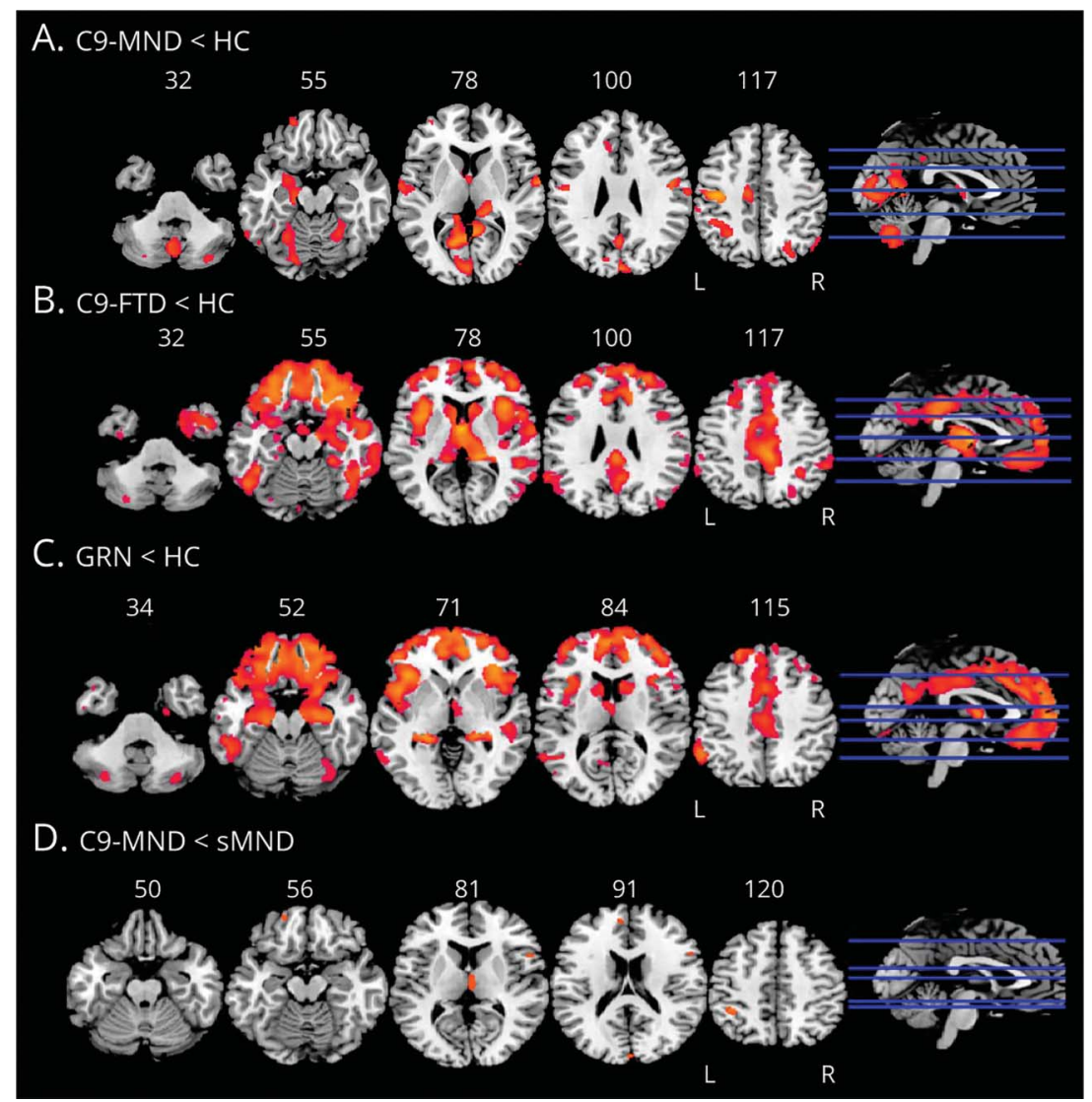

Results of voxel-based morphometry analysis showing regions of significant GM atrophy in FTLD genetic subgroups when compared with healthy controls ( $\mathrm{HCs})$, patients with sporadic disease, and between each other. Significant clusters are overlaid on sections of the Montreal Neurologic Institute standard brain. Analyses were corrected for age, sex and total intracranial volume. Statistical threshold for significance was $p<0.05$, family-wise error-corrected for multiple comparisons. C9-FTD = patients with frontotemporal dementia carrying a C9orf72 mutation; C9-MND = patients with motor neuron disease carrying a C9orf72 mutation; FTD = frontotemporal dementia; gFTD = genetic frontotemporal dementia; gMND = genetic motor neuron disease; $\mathrm{MND}=$ motor neuron disease; SFTD = sporadic frontotemporal dementia; $\mathrm{SMND}=$ sporadic motor neuron disease.

\section{Results}

\section{Genetic Findings}

The 66 FTLD mutation carriers (Figure 1A) showed pathogenic alterations of the following genes: C9orf72 $(n=33)$, TARDBP $(\mathrm{n}=10), \operatorname{GRN}(\mathrm{n}=8)$, SOD1 $(\mathrm{n}=7)$, FUS $(\mathrm{n}=2)$, TBK1 $(\mathrm{n}=2)$, and TREM2 $(\mathrm{n}=1)$; one patient showed both a C9orf72 expansion and a mutation of the GRN gene and one had both a C9orf72 and a TARDBP mutation.

\section{Clinical Diagnosis According to Genotype}

eTables 1 and 4 (available from Dryad, doi.org/10.5061/dryad. 1vhhmgqsz) report the clinical diagnoses of patients with gFTLD, according to the identified mutations. We found that each mutation was specifically associated with either an FTD (as for GRN, MAPT, and TREM2) or an MND presentation (as for TARDBP, SOD1, TBK1, and FUS), with the notable exception of patients with a C9orf72 mutation, 22 of which presented with MND (C9-MND) and 11 with FTD (C9-FTD). Patients carrying a double mutation (i.e., the C9orf $72+G R N$ and $C 9$ orf $72+$ TARDBP cases) presented, respectively, with FTD and MND.

\section{Clinical and Sociodemographic Features}

Sociodemographic and clinical characteristics of groups defined by clinical diagnosis and genetic status are reported in
Table 1. Groups were comparable in terms of sex, education, age at MRI and disease duration at MRI, and specific measures of disease severity for FTD or MND.

When assessing the most sizeable gFTLD subgroups, according to underlying mutation (i.e., C9-MND, C9-FTD, SOD1, $T A R D B P$, and $G R N$ ), a faster disease progression rate was found in patients with TARDBP as compared to sMND, C9-MND, and SOD1 (eTable 1 available from Dryad doi.org/10.5061/ dryad.1vhhmgqsz).

\section{Neuropsychological Features}

eTable 2, available from Dryad (doi.org/10.5061/dryad. 1vhhmgqsz), reports the neuropsychological test scores of groups defined by clinical diagnosis and genetic status. Patients with sFTD and patients with gFTD showed significant impairment of memory, executive, and linguistic functions. Notably, only patients with gFTD had significant visuospatial impairment, compared with healthy controls. Overall, neuropsychological features of MND groups were comparable with healthy controls, although 9 patients with sMND and 10 patients with gMND met criteria for mild cognitive or behavioral impairment. When comparing gMND and gFTD with the relative sporadic groups, no significant differences were detected. 
Figure 5 Gray Matter (GM) Volumes of Subcortical and Cerebellar Structures in Patients With Frontotemporal Lobar Degeneration (FTLD) According to Genetic Status and Clinical Presentation

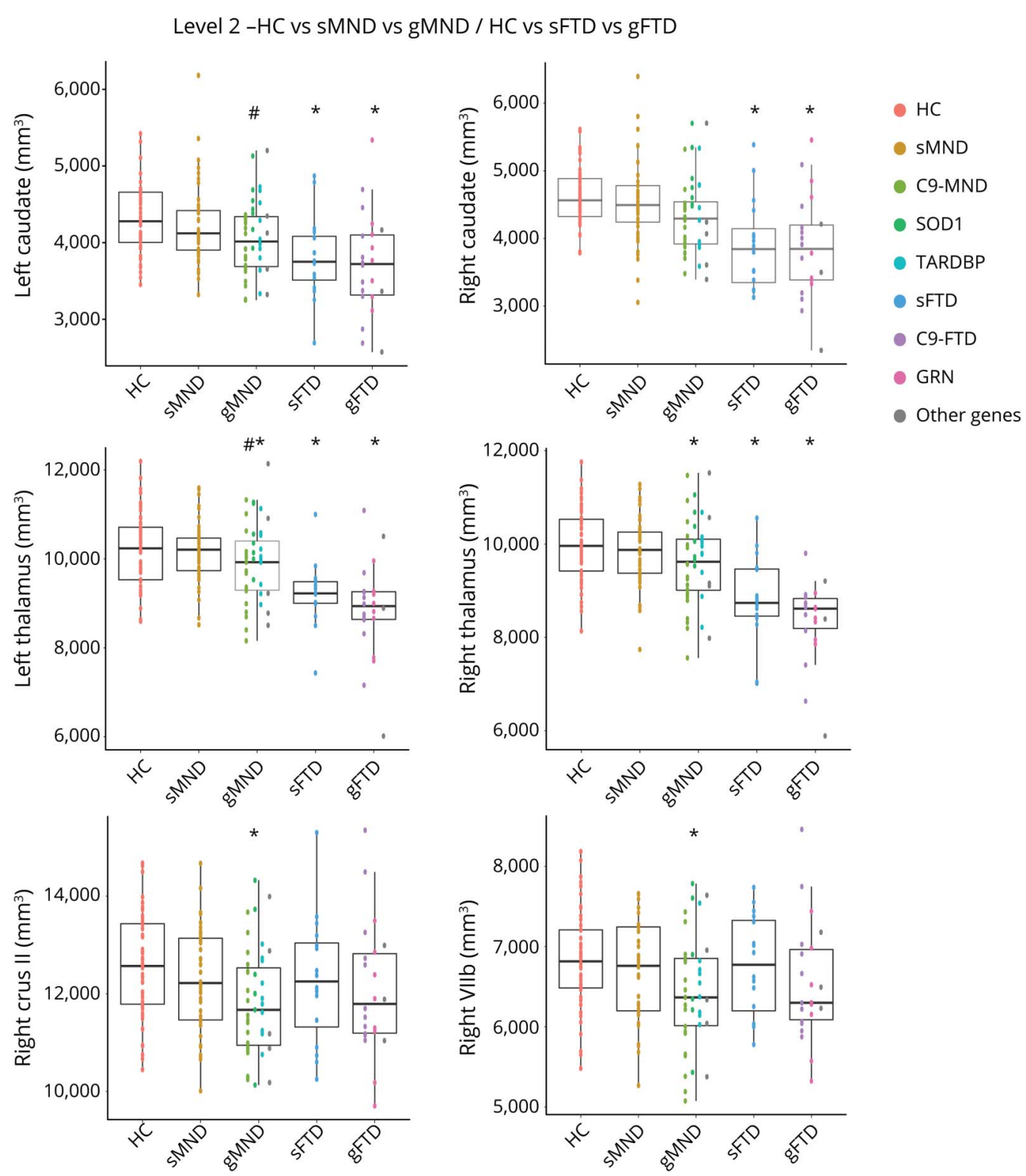

Comparisons between groups were made using age-, sex- and MRI scanner-adjusted analysis of variance models, followed by post hoc pairwise comparisons, Bonferroni-corrected for multiple comparisons. * $p<0.05$ compared with healthy controls (HCs); $\# p<0.05$ compared with sporadic motor neuron disease (SMND). C9-FTD = patients with frontotemporal dementia carrying a C9orf72 mutation; C9-MND = patients with motor neuron disease carrying a C9orf72 mutation; FTD = frontotemporal dementia; MND = motor neuron disease; SFTD = sporadic frontotemporal dementia.

eTables 3 and 4, available from Dryad (doi.org/10.5061/ dryad.1vhhmgqsz), report detailed neuropsychological features according to the underlying mutation. Of note, GRN mutation carriers showed the greatest visuospatial impairment, although this was not statistically significant compared with other groups.

\section{GM Atrophy}

For both VBM and volumetric analyses, characteristic patterns of GM atrophy in gFTLD were investigated using 3 different levels of comparison, following a systematic scheme that would allow an unbiased reading and interpretation of results (Figure 1B). First, 3 broad groups were compared: gFTLD, sFTLD, and healthy controls (level 1). As a second step, patients with FTLD were subdivided according to genetic status and phenotypic manifestation, so that sMND, sFTD, gMND, and gFTD were considered separately (level 2). Finally, for sufficiently sized genotypic groups, a further subdivision according to specific mutations was considered (level 3). Considering the clinical rationale of the study, aiming at describing variability of atrophy according to the genetic background, and the expected greater atrophy in FTD 
Figure 6 Relationship Between Thalamic Volume and Frontal Behavioral Inventory (FBI) Total Scores in Patients With Frontotemporal Lobar Degeneration (FTLD)
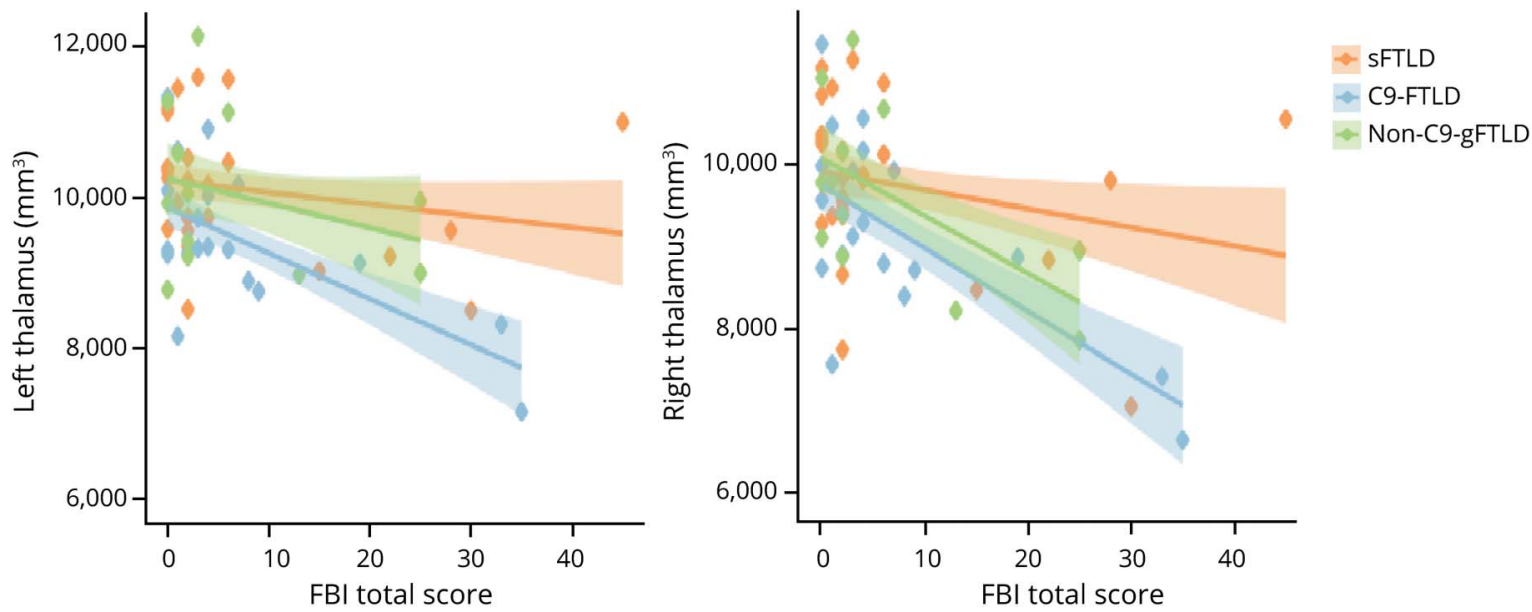

Plots showing significant inverse correlation only in patients with FTLD carrying a C9orf72 mutation (C9-FTLD) group (left thalamus: $r=-0.579, p=0.024$; right thalamus: $r=-0.613, p=0.025)$. Orange dots represent plotted values of patients with sporadic FTLD (sFTLD) ( $n=26$, based on availability of FBI scores), blue dots represent patients with C9-FTLD $(n=19)$, and green dots represent patients with genetic FTLD (gFTLD) with mutations other than C9orf72 ( $=12)$. Partial correlation analyses were Bonferroni-corrected for multiple comparisons, adjusted for age, sex, and education.

compared with MND, comparisons between sporadic/ genetic cases and healthy controls were performed separately for MND and FTD presentations for levels 2 and 3.

\section{Voxel-Based Morphometry}

\section{Level 1 (gFTLD vs sFTLD vs Controls)}

Compared with healthy controls, patients with sFTLD and patients with gFTLD showed significant GM volume loss of the prefrontal, anterior cingulate, insular and anterior temporal cortical regions, hippocampi, caudate nuclei, and cerebellar crus II, bilaterally (Figure 2, A and B; eTable 5, available at Dryad, doi.org/10.5061/dryad.1vhhmgqsz); in addition, patients with gFTLD showed a more widespread pattern of atrophy, also including the angular gyri, posterior temporal and posterior cingulate cortices, and thalami (Figure 2B). When compared directly with sFTLD, patients with gFTLD showed greater GM atrophy of the left angular gyrus (Figure 2C). No regions showing significantly greater atrophy in patients with sFTLD compared with patients with gFTLD were found.

\section{Level 2 (gMND vs sMND vs Controls; gFTD vs sFTD vs Controls)}

Compared with healthy controls, patients with sMND showed selective atrophy of the left precentral cortex (Figure 3A, eTable 6, available at Dryad, doi.org/10.5061/dryad.1vhhmgqsz); patients with gMND showed atrophy of the left hippocampus, angular gyrus, occipital cortex, Rolandic operculum, left crus II, cerebellar vermis VIIIa, and lobule VIIb, bilaterally (Figure 3B). Compared with sMND, patients with gMND showed greater atrophy of the left superior frontal and angular gyri (Figure 3E).

Compared with controls, patients with sFTD showed widespread atrophy of the prefrontal, insular, anterior temporal and anterior cingulate cortical regions, caudate nuclei, and cerebellar crus I, bilaterally (Figure 3C); patients with gFTD showed atrophy of similar regions, with the additional involvement of the thalami, posterior cingulate, fusiform and angular gyri bilaterally, left middle temporal gyrus, and right primary sensory cortex (Figure 3D). Compared with sFTD, patients with gFTD showed greater GM atrophy of the thalami and superior parietal lobules, bilaterally, left angular gyrus, and right posterior cingulate cortex (Figure 3F).

No regions showing significantly greater atrophy in sporadic MND/FTD compared with genetic MND/FTD were found.

\section{Level 3 (C9-MND vs SOD1 vs TARDBP vs sMND vs Controls; C9- FTD vs GRN vs sFTD vs Controls)}

Compared with healthy controls, patients with C9-MND showed atrophy of the left precentral and postcentral gyri, left supplementary motor area, right angular gyrus, and right occipital cortex, as well as the left thalamus and cerebellar vermis lobule V and lobule VIIb, bilaterally (Figure 4A, eTable 7, available at Dryad doi.org/10.5061/dryad.1vhhmgqsz); patients with MND with other mutations (i.e., SOD1 and TARDBP mutation carriers) did not show significant GM atrophy. Compared with patients with sMND, patients with C9-MND showed greater atrophy of the inferior frontal gyri, bilaterally, left postcentral gyrus, and right posterior thalamus (Figure 4D). No other statistically significant differences between MND groups were found.

Patients with C9-FTD showed widespread bilateral atrophy of the prefrontal, insular, and cingulate cortices, angular gyri, caudate nuclei, thalami, and cerebellar left crus II and vermis lobule IX (Figure 4B); patients with GRN showed atrophy of 
the prefrontal, insular, and anterior cingulate cortices, hippocampi, bilaterally, left angular and fusiform gyri, caudate nuclei, thalami, and cerebellar crus II, bilaterally (Figure 4C). Although no statistically significant differences between FTD groups were found, it is worth noting that thalamic involvement in C9-FTD was more widespread, involving both anterior and posterior regions, if visually compared with the selective damage of anterior thalamic regions observed in GRN cases.

\section{Volumetric GM Analysis}

\section{Level 1 (gFTLD vs sFTLD vs Controls)}

Compared with healthy controls, patients with sFTLD showed decreased volume of the right thalamus, left putamen, left amygdala, and left hippocampus; patients with gFTLD showed volumetric reduction of the caudate nuclei and thalami, bilaterally, left putamen, left hippocampus, right cerebellar crus II, and right cerebellar lobule VIIb (eFigure 1 and eTable 8, available from Dryad, doi.org/10.5061/dryad. 1vhhmgqsz). Compared with sFTLD, patients with gFTLD showed volumetric reduction of the thalami, bilaterally, right caudate, and right cerebellar lobule VIIb.

\section{Level 2 (gMND vs sMND vs Controls; gFTD vs sFTD vs Controls)}

Compared with healthy controls, patients with sMND did not show any significant volumetric reduction of the considered GM structures, whereas patients with gMND showed decreased thalamic volumes, bilaterally, and greater atrophy of the right cerebellar crus II and right lobule VIIb (Figure 5, eTable 9, available from Dryad, doi.org/10.5061/dryad. 1vhhmgqsz). Compared with patients with sMND, patients with gMND showed volumetric reduction of the left caudate and left thalamus, and a trend toward greater atrophy of the right crus II $(p=0.07)$.

Compared with controls, patients with sFTD showed decreased volume of the caudate nuclei, thalami, hippocampi, putamina, and pallidi, bilaterally, and left amygdala; gFTD showed similar widespread volume loss of the caudate nuclei, thalami, hippocampi, putamina, and pallidi, bilaterally, as well as a trend toward greater atrophy of the right cerebellar lobule VIIb $(p=0.067)$ (Figure 5, eTable 9, available from Dryad, doi.org/10.5061/dryad.1vhhmgqsz). No significant differences between sFTD and gFTD were found.

\section{Level 3 (C9-MND vs SOD1 vs TARDBP vs sMND vs Controls; C9-FTD vs GRN vs sFTD vs Controls)}

Compared with healthy controls, patients with C9-MND showed significant volumetric reduction of the caudate nuclei, thalami, cerebellar lobules VIIb, bilaterally, and right crus II (eFigure 2, eTables 10-11, available from Dryad, doi.org/10. 5061/dryad.1vhhmgqsz). Compared with patients with sMND, patients with C9-MND showed significant volumetric reduction of the thalami, bilaterally, and left caudate, and a trend toward greater atrophy of the right cerebellar lobule VIIb $(p=0.07)$.

Compared with healthy controls, patients with C9-FTD showed volumetric reduction of the caudate nuclei and thalami, bilaterally; patients with GRN showed volumetric reduction of the caudate nuclei, thalami, and hippocampi, bilaterally, and left putamen (eFigure 2, eTables 10-11, available from Dryad, doi.org/10.5061/dryad.1vhhmgqsz). Compared with patients with sFTD, no statistically significant differences of MRI volumetric measures of patients with C9FTD and patients with GRN were found.

\section{Correlations Between Clinical, Cognitive, and MRI Volumes of Patients With FTLD}

Thalamic volumes of patients with gFTLD showed an inverse correlation with Frontal Behavioral Inventory total scores (left: $\mathrm{r}=-0.473, p=0.031$; right: $\mathrm{r}=-0.638, p=0.002$ ). After subdividing gFTLD according to C9orf72 status (i.e., C9FTLD and non-C9 gFTLD), this correlation remained significant only for C9-FTLD (left: $\mathrm{r}=-0.579, p=0.024$; right: $\mathrm{r}=-0.613, p=0.025$ ) (Figure 6).

No other statistically significant correlations between clinical/ cognitive and MRI volumetric features of patients with FTLD were found.

\section{Discussion}

The present study provides a comprehensive report of clinical and GM structural MRI findings in a cohort of patients with disorders of the FTLD spectrum with known genetic mutations. Patients with genetically determined FTLD (i.e., both FTD and MND presentations) consistently showed greater GM disruption, compared with sporadic cases who were matched for clinical presentation and degree of functional and cognitive impairment. In particular, the involvement of parietal cortices, thalami, and cerebellar regions was observed consistently in gFTLD cases, in contrast with sFTD showing atrophy mostly affecting fronto-temporo-insular regions and basal ganglia, and sMND displaying focal damage of motor cortical regions. We have described distinctive patterns of atrophy that associate with each specific mutation, identifying the reduction of thalamic volumes as mostly indicative of C9orf72-mutated cases, in particular for patients presenting with MND. The results provide interesting insights into the pathophysiology of gFTLD and suggest possible neuroimaging markers of underlying pathology that may help to disentangle the heterogeneity of disorders of the FTLD spectrum.

Some important observations can be drawn regarding the relationship between clinical phenotype and the underlying genetic background in the present sample. Our gFTLD cohort included a prevalent proportion of MND cases, compared with previous studies, which were mostly focused on 
FTD. $^{7,8,11}$ Only studies assessing C9orf72 mutation carriers $^{24,25}$ had a larger representation of MND, consistent with the known association of this mutation with both cognitive and motor FTLD presentations. In fact, in our cohort, C9orf72 was the only mutation that was observed both in FTD and MND cases, and all patients presenting with a mixed FTD-ALS phenotype carried this genetic alteration. The composition of our cohort also allowed the identification of relatively sizeable groups of less common MND-related mutation carriers, whose neuroanatomical damage has been rarely described, such as $S O D 1^{26,27}$ or TARDBP. ${ }^{28,29}$ In addition, we highlighted that not only ALS, but also other MND phenotypes (i.e., PLS and PMA) can be associated with C9orf72, TARDBP, or SOD1 mutations. PLS/PMA cases with a C9orf72 expansion have been rarely described, ${ }^{30}$ whereas an association with $S O D 1$ or TARDBP mutations is practically anecdotic. $^{31}$ Therefore, our findings suggest that the common notion of PLS and PMA as sporadic MND presentations should be at least reconsidered. ${ }^{31}$

The sporadic groups of our sample were selected to be matched with gFTLD cases for clinical presentation and disease severity. Consistently, gMND and gFTD were comparable with the respective sporadic groups in terms of the main clinical and cognitive measures. A notable exception was provided by TARDBP mutation carriers, whose disease progression rate was faster than other MND groups, although with a high interindividual variability, consistent with previous reports. ${ }^{32}$ Although we did not detect significant differences in neuropsychological measures between genetic FTD/MND and the respective sporadic groups, gFTD (in particular, GRN mutation carriers) had the most severe impairment of visuospatial skills, suggesting a more rapid evolution to multidomain cognitive impairment, similar to previous reports in GRN mutation carriers. ${ }^{33}$

The most consistent result provided by VBM was more severe and widespread GM atrophy in patients with gFTLD compared with sFTLD. We revealed a characteristic involvement of the inferior parietal, posterior cingulate, thalamic, and posterior cerebellar regions in gFTD and gMND that the respective sporadic groups lacked. GM atrophy was generally greater and more diffuse in patients with gFTD, followed by sFTD and gMND cases, whereas patients with sMND showed very focal, subtle atrophy of the motor cortex (which was shared by patients with gMND). The presence of diffuse neuroanatomical damage in gFTLD compared with sFTLD, extending to posterior cortical and subcortical regions despite a comparable disease severity and duration, supports the notion that an unfavorable genetic background might accelerate neurodegeneration in neuronal populations that are relatively distant from those classically involved in FTLD. ${ }^{34,35}$

When we assessed patients defined on the specific underlying mutation, a greater damage of the inferior parietal cortices (namely, the angular gyrus) and the thalami was shared by C9MND, C9-FTD, and GRN mutation carriers, compared with sporadic cases. The greater involvement of the inferior parietal regions is consistent with previous reports in C9orf $72^{24,36-38}$ and GRN mutation carriers, ${ }^{1,33}$ even from the presymptomatic stages. ${ }^{37,38}$ Patients with GRN showed a left-sided prevalence of parietal cortical damage, consistent with the relatively large proportion (3/8) of PPA presentations in our cohort and the known asymmetrical atrophy of this group, ${ }^{1,33}$ although the overall average pattern of GM atrophy was relatively symmetrical. Of note, characteristic posterior thalamic and cerebellar atrophy was found in C9orf 72 mutation carriers with either FTD or MND presentations, in contrast with the involvement of anterior thalamic regions in GRN mutation carriers. Our findings are in line with previous studies highlighting the specific involvement of the pulvinar and posterior cerebellar regions in FTD cases with a C9orf72 expansion, and expanding the validity of these findings to pure MND cases. ${ }^{7,8,11}$ By contrast, we were not able to detect significant atrophy in the cortical regions of TARDBP and SOD1 cases, although these groups were similarly sized when compared with C9-MND. To our knowledge, there are no published reports of MRI volumetric findings in a TARDBP cohort, due to the rarity of this mutation. Although our findings need to be confirmed in larger samples, these suggest a substantial absence of GM involvement in patients with MND carrying a TARDBP mutation, in contrast with the few case reports demonstrating frontotemporal atrophy in TARDBP-related FTD cases. ${ }^{28,29}$ The absence of brain GM atrophy in our SOD1 group is in line with studies suggesting a different distribution of the non-TDP43 pathology associated with this mutation ${ }^{26,27}$ that prevalently involves the lower motor neurons in the spinal cord. ${ }^{39}$

Intrigued by the results that we obtained at the whole-brain level, we then focused on the involvement of deep GM and cerebellar structures, in order to identify quantitative volumetric markers that could provide relevant group-specific measures of neurodegeneration in gFTLD. In this case, the difference in severity of atrophy between MND and FTD was even more relevant compared with the VBM analysis, since both sFTD and gFTD showed a similarly widespread severe involvement of basal ganglia, thalami, and hippocampi, in contrast with a general preservation of these structures in sMND and gMND. The only notable exception was the significant bilateral thalamic atrophy identified in gMND that emerged particularly when only C9-MND were considered, as a distinctive feature compared with sMND cases. In fact, our findings strengthen previous evidence that thalamic atrophy in FTLD is highly indicative of a genetic underlying cause, mostly pointing toward a C9orf72 expansion. ${ }^{7,24,40,41}$ We have also shown distinctive atrophy of the caudate nucleus in C9MND cases, consistent with previous MRI studies demonstrating typical basal ganglia involvement in C9orf72-related $\mathrm{MND}^{10}$ and subtle functional rearrangements in the thalami and basal ganglia of C9orf72 mutation carriers, even in presymptomatic phases. ${ }^{42,43} \mathrm{We}$ have also found an inverse correlation between thalamic volumes and behavioral impairment in patients with gFTLD, which was mostly driven by C9orf72 mutation carriers. This suggests a significant 
influence of such characteristic neuroanatomical damage over the progression of neurobehavioral impairment in C9orf72related FTLD, consistent with the involvement of the thalami in cognition and complex behavior. ${ }^{44}$ Therefore, our results strongly point towards the use of measures of deep GM involvement as useful markers of C9orf72-related disorders, regardless of the clinical presentation within the FTLD spectrum.

The analysis of cerebellar volumes showed a substantial preservation of these regions in sFTLD, in contrast with atrophy of the lobule VIIb and crus II detected in gFTLD. Particularly, the single cerebellar structure showing greater damage in gFTLD compared with sFTLD was the right lobule VIIb. When looking at single genetic alterations, we identified gMND and, particularly, C9-MND as driving these results, which are consistent with recent studies indicating the involvement of the cognitive/affective regions of the posterior cerebellum (particularly, lobule VII and crus regions, involved in the modulation of emotions and social behaviors) as indicative of the presence of a $C 9$ orf 72 mutation, ${ }^{7}$ possibly as a consequence of the close structural and functional connections with the thalami through cerebello-thalamo-cortical networks. ${ }^{42,45}$ Similar alterations have been demonstrated in presymptomatic C9orf 72 mutation carriers, ${ }^{37,38,46}$ correlating with cognitive inhibition deficits. ${ }^{46}$ Although the role in cognition and the topologic organization of the cerebellar cortex has started being elucidated only in recent years, ${ }^{45}$ this is an exciting area of developing research for biomarkers of disease pathology in the FTLD spectrum (including MND) that might be combined with other more established measures of cortical damage.

This study is not without limitations. First, in order to include the largest possible number of gFTLD cases, we included cases acquired using 2 different MRI scanners. For this reason, we corrected all analyses for scanner type. Moreover, we lacked neuropathologic postmortem diagnosis of the sporadic cases. This might have partially influenced the results when comparing cases of sFTD with gFTD, which, in our cohort, were almost exclusively due to mutations leading to FTLDTDP pathology. Finally, we did not involve presymptomatic mutation carriers, which would be needed to understand how early the volumetric changes that we detected can be observed in the course of the disease. Future longitudinal studies involving these cases will be fundamental to understand the potential clinical relevance of these measures for early identification of patients close to symptom onset and as outcome measures in clinical trials targeting specific FTLD-related molecular mechanisms.

This study encompassed the entire FTLD spectrum, providing an accurate overview of clinical, neuropsychological, and MRI volumetric findings in patients with a genetically determined FTD/MND clinical phenotype. We also described mutations that have been rarely reported previously in the neuroimaging literature and identified specific imaging measures of FTLD genotypes, which proved especially useful when it comes to C9orf72-associated presentations. Our results strongly suggest that neuroimaging can provide useful volumetric measures applicable to future clinical trials targeting these genetic mutations.

\section{Acknowledgment}

The authors thank the patients and their families for participation and Laura Pozzi for assistance with genetic data analysis.

\section{Study Funding}

Italian Ministry of Health (RF-2011-02351193; GR-201102351217; GR-2013-02357415) and the European Research Council (StG-2016_714388_NeuroTRACK).

\section{Disclosure}

E.G. Spinelli, A. Ghirelli, S. Basaia, C. Cividini, and N. Riva have nothing to disclose. E. Canu receives or has received research support from the Italian Ministry of Health. V. Castelnovo, T. Domi, G. Magnani, F. Caso, P. Caroppo, S. Prioni, G. Rossi, L. Tremolizzo, and I. Appollonio have nothing to disclose. V. Silani received compensation for consulting services and/or speaking activities from AveXis, Cytokinetics, and Italfarmaco and receives or has received research supports from the Italian Ministry of Health, AriSLA, and E-Rare Joint Transnational Call. P. Carrera has nothing to disclose. M. Filippi is Editor-in-Chief of the Journal of Neurology and Associate Editor of Human Brain Mapping, received compensation for consulting services and/or speaking activities from Almiral, Alexion, Bayer, Biogen, Celgene, Eli Lilly, Genzyme, Merck-Serono, Novartis, Roche, Sanofi, Takeda, and Teva Pharmaceutical Industries, and receives research support from Biogen Idec, Merck-Serono, Novartis, Roche, Teva Pharmaceutical Industries, Italian Ministry of Health, Fondazione Italiana Sclerosi Multipla, and ARiSLA (Fondazione Italiana di Ricerca per la SLA). F. Agosta is Associate Editor of NeuroImage: Clinical, has received speaker honoraria from Novartis, Biogen Idec, and Philips, and receives or has received research supports from the Italian Ministry of Health, AriSLA (Fondazione Italiana di Ricerca per la SLA), and the European Research Council. Go to Neurology.org/ $\mathrm{N}$ for full disclosures.

\section{Publication History}

Received by Neurology March 10, 2021. Accepted in final form August 9, 2021.

Appendix Authors

\begin{tabular}{lll}
\hline Name & Location & Contribution \\
\hline $\begin{array}{l}\text { Edoardo G. } \\
\text { Spinelli, MD }\end{array}$ & $\begin{array}{l}\text { Neuroimaging Research } \\
\text { Unit, Division of } \\
\text { Neuroscience, IRCCS San } \\
\text { Raffaele Scientific Institute, } \\
\text { and Vita-Salute San Raffaele } \\
\text { University, Milan, Italy }\end{array}$ & $\begin{array}{l}\text { Design and } \\
\text { conceptualization of study; } \\
\text { acquisition, statistical } \\
\text { analysis, and interpretation } \\
\text { the manuscript for } \\
\text { intellectual content }\end{array}$ \\
& & \\
\hline
\end{tabular}


Appendix (continued)

\begin{tabular}{|c|c|c|}
\hline Name & Location & Contribution \\
\hline $\begin{array}{l}\text { Alma } \\
\text { Ghirelli, MD }\end{array}$ & $\begin{array}{l}\text { Neuroimaging Research } \\
\text { Unit, Division of } \\
\text { Neuroscience, IRCCS San } \\
\text { Raffaele Scientific Institute, } \\
\text { and Vita-Salute San Raffaele } \\
\text { University, Milan, Italy }\end{array}$ & $\begin{array}{l}\text { Acquisition, statistical } \\
\text { analysis, and interpretation } \\
\text { of data; drafted and revised } \\
\text { the manuscript for } \\
\text { intellectual content }\end{array}$ \\
\hline $\begin{array}{l}\text { Silvia } \\
\text { Basaia, PhD }\end{array}$ & $\begin{array}{l}\text { Neuroimaging Research } \\
\text { Unit, Division of } \\
\text { Neuroscience, IRCCS San } \\
\text { Raffaele Scientific Institute, } \\
\text { Milan, Italy }\end{array}$ & $\begin{array}{l}\text { Acquisition, statistical } \\
\text { analysis, and interpretation } \\
\text { of data; drafted and revised } \\
\text { the manuscript for } \\
\text { intellectual content }\end{array}$ \\
\hline $\begin{array}{l}\text { Camilla } \\
\text { Cividini, } \\
\text { MSc }\end{array}$ & $\begin{array}{l}\text { Neuroimaging Research } \\
\text { Unit, Division of } \\
\text { Neuroscience, IRCCS San } \\
\text { Raffaele Scientific Institute, } \\
\text { and Vita-Salute San Raffaele } \\
\text { University, Milan, Italy }\end{array}$ & $\begin{array}{l}\text { Acquisition, statistical } \\
\text { analysis, and interpretation } \\
\text { of data; drafted and revised } \\
\text { the manuscript for } \\
\text { intellectual content }\end{array}$ \\
\hline
\end{tabular}

Nilo Riva, Unit of Neurorehabilitation,

MD, PhD and Experimental interpretation of data;

Neuropathology Unit, IRCCS revised the manuscript for

San Raffaele Scientific intellectual content

Institute, Milan, Italy

\begin{tabular}{|c|c|c|}
\hline $\begin{array}{l}\text { Elisa Canu, } \\
\text { PhD }\end{array}$ & $\begin{array}{l}\text { Neuroimaging Research } \\
\text { Unit, Division of } \\
\text { Neuroscience, IRCCS San } \\
\text { Raffaele Scientific Institute, } \\
\text { Milan, Italy }\end{array}$ & $\begin{array}{l}\text { Acquisition and } \\
\text { interpretation of data; } \\
\text { drafted and revised the } \\
\text { manuscript for intellectual } \\
\text { content }\end{array}$ \\
\hline $\begin{array}{l}\text { Veronica } \\
\text { Castelnovo, } \\
\text { MSc }\end{array}$ & $\begin{array}{l}\text { Neuroimaging Research } \\
\text { Unit, Division of } \\
\text { Neuroscience, IRCCS San } \\
\text { Raffaele Scientific Institute, } \\
\text { and Vita-Salute San Raffaele } \\
\text { University, Milan, Italy }\end{array}$ & $\begin{array}{l}\text { Acquisition, statistical } \\
\text { analysis, and interpretation } \\
\text { of data; drafted and revised } \\
\text { the manuscript for } \\
\text { intellectual content }\end{array}$ \\
\hline
\end{tabular}

\begin{tabular}{ll}
\hline $\begin{array}{l}\text { Teuta Domi, } \\
\text { PhD }\end{array}$ & $\begin{array}{l}\text { Experimental } \\
\text { Neuropathology Unit, IRCCS } \\
\text { San Raffaele Scientific } \\
\text { Institute, Milan, Italy }\end{array}$ \\
\hline $\begin{array}{l}\text { Giuseppe } \\
\text { Magnani, } \\
\text { MD }\end{array}$ & $\begin{array}{l}\text { Unit of Neurology, IRCCS San } \\
\text { Raffaele Scientific Institute, } \\
\text { Milan, Italy }\end{array}$
\end{tabular}

Acquisition and

interpretation of data;

revised the manuscript for intellectual content

Acquisition and

interpretation of data; revised the manuscript for intellectual content

\begin{tabular}{ll}
\hline Francesca & Unit of Neurology, IRCCS San \\
Caso, MD, & Raffaele Scientific Institute, \\
PhD & Milan, Italy
\end{tabular}

Acquisition and

interpretation of data; revised the manuscript for intellectual content

\begin{tabular}{|c|c|c|}
\hline $\begin{array}{l}\text { Paola } \\
\text { Caroppo, } \\
\text { MD, PhD }\end{array}$ & 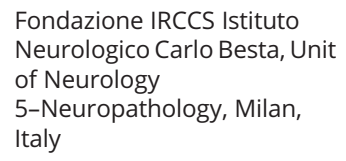 & $\begin{array}{l}\text { Acquisition and } \\
\text { interpretation of data; } \\
\text { revised the manuscript for } \\
\text { intellectual content }\end{array}$ \\
\hline $\begin{array}{l}\text { Sara Prioni, } \\
\text { MSc }\end{array}$ & $\begin{array}{l}\text { Fondazione IRCCS Istituto } \\
\text { Neurologico Carlo Besta, Unit } \\
\text { of Neurology } \\
\text { 5-Neuropathology, Milan, } \\
\text { Italy }\end{array}$ & $\begin{array}{l}\text { Acquisition and } \\
\text { interpretation of data; } \\
\text { revised the manuscript for } \\
\text { intellectual content }\end{array}$ \\
\hline $\begin{array}{l}\text { Giacomina } \\
\text { Rossi, PhD }\end{array}$ & $\begin{array}{l}\text { Fondazione IRCCS Istituto } \\
\text { Neurologico Carlo Besta, Unit } \\
\text { of Neurology } \\
\text { 5-Neuropathology, Milan, } \\
\text { Italy }\end{array}$ & $\begin{array}{l}\text { Acquisition and } \\
\text { interpretation of data; } \\
\text { revised the manuscript for } \\
\text { intellectual content }\end{array}$ \\
\hline $\begin{array}{l}\text { Lucio } \\
\text { Tremolizzo, } \\
\text { MD }\end{array}$ & $\begin{array}{l}\text { Neurology Unit, "San } \\
\text { Gerardo" Hospital and } \\
\text { University of Milano-Bicocca, } \\
\text { Monza, Italy }\end{array}$ & $\begin{array}{l}\text { Acquisition and } \\
\text { interpretation of data; } \\
\text { revised the manuscript for } \\
\text { intellectual content }\end{array}$ \\
\hline
\end{tabular}

Appendix (continued)

\begin{tabular}{|c|c|c|}
\hline Name & Location & Contribution \\
\hline $\begin{array}{l}\text { Ildebrando } \\
\text { Appollonio, } \\
\text { MD }\end{array}$ & $\begin{array}{l}\text { Neurology Unit, "San } \\
\text { Gerardo" Hospital and } \\
\text { University of Milano-Bicocca, } \\
\text { Monza, Italy }\end{array}$ & $\begin{array}{l}\text { Acquisition and } \\
\text { interpretation of data; } \\
\text { revised the manuscript for } \\
\text { intellectual content }\end{array}$ \\
\hline $\begin{array}{l}\text { Vincenzo } \\
\text { Silani, MD }\end{array}$ & $\begin{array}{l}\text { Department of Neurology } \\
\text { and Laboratory of } \\
\text { Neuroscience, IRCCS Istituto } \\
\text { Auxologico Italiano, Milan, } \\
\text { Italy }\end{array}$ & $\begin{array}{l}\text { Acquisition and } \\
\text { interpretation of data; } \\
\text { revised the manuscript for } \\
\text { intellectual content }\end{array}$ \\
\hline $\begin{array}{l}\text { Paola } \\
\text { Carrera, BSc }\end{array}$ & $\begin{array}{l}\text { Laboratory of Clinical } \\
\text { Molecular Biology, IRCCS San } \\
\text { Raffaele Scientific Institute, } \\
\text { Milan, Italy }\end{array}$ & $\begin{array}{l}\text { Acquisition and } \\
\text { interpretation of data; } \\
\text { revised the manuscript for } \\
\text { intellectual content }\end{array}$ \\
\hline $\begin{array}{l}\text { Massimo } \\
\text { Filippi, MD }\end{array}$ & $\begin{array}{l}\text { Neuroimaging Research } \\
\text { Unit, Division of } \\
\text { Neuroscience, Unit of } \\
\text { Neurorehabilitation, Unit of } \\
\text { Neurology, Neurophysiology } \\
\text { Service, IRCCS San Raffaele } \\
\text { Scientific Institute, and Vita- } \\
\text { Salute San Raffaele } \\
\text { University, Milan, Italy }\end{array}$ & $\begin{array}{l}\text { Design and } \\
\text { conceptualization of study; } \\
\text { interpretation of data; } \\
\text { revised the manuscript for } \\
\text { intellectual content; } \\
\text { obtained funding }\end{array}$ \\
\hline $\begin{array}{l}\text { Federica } \\
\text { Agosta, MD, } \\
\text { PhD }\end{array}$ & $\begin{array}{l}\text { Neuroimaging Research } \\
\text { Unit, Division of } \\
\text { Neuroscience, Unit of } \\
\text { Neurology, IRCCS San } \\
\text { Raffaele Scientific Institute; } \\
\text { and Vita-Salute San Raffaele } \\
\text { University, Milan, Italy }\end{array}$ & $\begin{array}{l}\text { Design and } \\
\text { conceptualization of study; } \\
\text { interpretation of data; } \\
\text { drafted and revised the } \\
\text { manuscript for intellectual } \\
\text { content; obtained funding }\end{array}$ \\
\hline
\end{tabular}

\section{References}

1. Ferrari R, Manzoni C, Hardy J. Genetics and molecular mechanisms of frontotemporal lobar degeneration: an update and future avenues. Neurobiol Aging. 2019;78: 98-110.

2. Filippi M, Agosta F. MRI of non-Alzheimer's dementia: current and emerging knowledge. Curr Opin Neurol. 2018;31(4):405-414.

3. Rascovsky K, Hodges JR, Knopman D, et al. Sensitivity of revised diagnostic criteria for the behavioural variant of frontotemporal dementia. Brain. 2011;134(9) 2456-2477.

4. Gorno-Tempini ML, Hillis AE, Weintraub S, et al. Classification of primary progressive aphasia and its variants. Neurology. 2011;76(11):1006-1014.

5. Elahi FM, Miller BL. A clinicopathological approach to the diagnosis of dementia. Nat Rev Neurol. 2017;13(8):457-476.

6. Cash DM, Bocchetta M, Thomas DL, et al. Patterns of gray matter atrophy in genetic frontotemporal dementia: results from the GENFI study. Neurobiol Aging. 2018;62: 191-196.

7. Bocchetta M, Iglesias JE, Neason M, Cash DM, Warren JD, Rohrer JD. Thalamic nuclei in frontotemporal dementia: mediodorsal nucleus involvement is universal but pulvinar atrophy is unique to C9orf72. Hum Brain Mapp. 2020;41(4):1006-1016.

8. Bocchetta M, Cardoso MJ, Cash DM, Ourselin S, Warren JD, Rohrer JD. Patterns of regional cerebellar atrophy in genetic frontotemporal dementia. Neuroimage Clin 2016;11:287-290.

9. Whitwell JL, Josephs KA. Neuroimaging in frontotemporal lobar degeneration predicting molecular pathology. Nat Rev Neurol. 2012;8(3):131-142.

10. Bede P, Omer T, Finegan E, et al. Connectivity-based characterisation of subcortical grey matter pathology in frontotemporal dementia and ALS: a multimodal neuroimaging study. Brain Imaging Behav. 2018;12(6):1696-1707.

11. Whitwell JL, Boeve BF, Weigand SD, et al. Brain atrophy over time in genetic and sporadic frontotemporal dementia: a study of 198 serial magnetic resonance images. Eur J Neurol. 2015;22(5):745-752.

12. Brooks BR, Miller RG, Swash M, Munsat TL. El Escorial revisited: revised criteria for the diagnosis of amyotrophic lateral sclerosis. Amyotroph Lateral Scler Other Mot Neuron Disord. 2000;1(5):293-299.

13. Van den Berg-Vos RM, Visser J, Franssen H, et al. Sporadic lower motor neuron disease with adult onset: classification of subtypes. Brain. 2003;126(5):1036-1047.

14. Pringle CE, Hudson AJ, Munoz DG, Kiernan JA, Brown WF, Ebers GC. Primary lateral sclerosis. Clinical features, neuropathology and diagnostic criteria. Brain. 1992; 115 (pt 2):495-520.

15. Knopman DS, Kramer JH, Boeve BF, et al. Development of methodology for conducting clinical trials in frontotemporal lobar degeneration. Brain. 2008;131(11): 2957-2968. 
Cedarbaum JM, Stambler N, Malta E, et al. The ALSFRS-R: a revised ALS functional rating scale that incorporates assessments of respiratory function. J Neurol Sci. 1999; 169(1-2):13-21.

17. Santangelo R, Dell'Edera A, Sala A, et al. The CSF p-tau181/A 342 ratio offers a good accuracy "in vivo" in the differential diagnosis of Alzheimer's dementia. Curr Alzheimer Res. 2019;16(7):587-595.

18. Spinelli EG, Agosta F, Ferraro PM, et al. Brain MRI shows white matter sparing in Kennedy's disease and slow-progressing lower motor neuron disease. Hum Brain Mapp. 2019;40(10):3102-3112.

19. Strong MJ, Abrahams S, Goldstein LH, et al. Amyotrophic lateral sclerosis: frontotemporal spectrum disorder (ALS-FTSD): revised diagnostic criteria. Amyotroph Lateral Scler Frontotemporal Degener. 2017;18(3-4):153-174.

20. Ashburner J. A fast diffeomorphic image registration algorithm. Neuroimage. 2007, 38(1):95-113.

21. Filippi M, Basaia S, Canu E, et al. Changes in functional and structural brain connectome along the Alzheimer's disease continuum. Mol Psychiatry. 2020;25(1):230-239.

22. Diedrichsen J, Balsters JH, Flavell J, Cussans E, Ramnani N. A probabilistic MR atlas of the human cerebellum. Neuroimage. 2009;46(1):39-46.

23. Diedrichsen J, Maderwald S, Küper M, et al. Imaging the deep cerebellar nuclei: probabilistic atlas and normalization procedure. Neuroimage. 2011;54(3):1786-1794

24. Mahoney CJ, Beck J, Rohrer JD, et al. Frontotemporal dementia with the C9ORF72 hexanucleotide repeat expansion: clinical, neuroanatomical and neuropathological features. Brain. 2012;135(3):736-750.

25. Floeter MK, Danielian LE, Braun LE, Wu T. Longitudinal diffusion imaging across the C9orf72 clinical spectrum. J Neurol Neurosurg Psychiatry. 2018;89(1):53-60.

26. Agosta F, Spinelli EG, Marjanovic IV, et al. Unraveling ALS due to SOD1 mutation through the combination of brain and cervical cord MRI. Neurology. 2018;90(8): e707-e716.

27. Turner MR, Hammers A, Allsop J, et al. Volumetric cortical loss in sporadic and familial amyotrophic lateral sclerosis. Amyotroph Lateral Scler. 2007;8(6):343-347.

28. Borroni B, Bonvicini C, Alberici A, et al. Mutation within TARDBP leads to fron totemporal dementia without motor neuron disease. Hum Mutat. 2009;30(11): E974-E983.

29. Gelpi E, van der Zee J, Turon Estrada A, Van Broeckhoven C, Sanchez-Valle R. TARDBP mutation p.Ile383Val associated with semantic dementia and complex proteinopathy. Neuropathol Appl Neurobiol. 2014;40(2):225-230.

30. Van Rheenen W, Van Blitterswijk M, Huisman MHB, et al. Hexanucleotide repeat expansions in C9ORF72 in the spectrum of motor neuron diseases. Neurology. 2012; $79(9): 878-882$

31. van Blitterswijk M, Vlam L, van Es MA, et al. Genetic overlap between apparently sporadic motor neuron diseases. PLoS One. 2012;7(11):e48983.
32. Corcia P, Valdmanis P, Millecamps S, et al. Phenotype and genotype analysis in amyotrophic lateral sclerosis with TARDBP gene mutations. Neurology 2012;78(19): 1519-1526.

33. Beck J, Rohrer JD, Campbell T, et al. A distinct clinical, neuropsychological and radiological phenotype is associated with progranulin gene mutations in a large UK series. Brain. 2008;131(pt 3):706-720.

34. Schönecker S, Neuhofer C, Otto M, et al. Atrophy in the thalamus but not cerebellum is specific for C9orf72 FTD and ALS patients: an atlas-based volumetric MRI study. Front Aging Neurosci. 2018;10:45.

35. Agosta F, Ferraro PM, Riva N, et al. Structural and functional brain signatures of C9orf72 in motor neuron disease. Neurobiol Aging. 2017;57:206-219.

36. Boeve BF, Boylan KB, Graff-Radford NR, et al. Characterization of frontotempora dementia and/or amyotrophic lateral sclerosis associated with the GGGGCC repeat expansion in C9ORF72. Brain. 2012;135(3):765-783.

37. Bertrand A, Wen J, Rinaldi D, et al. Early cognitive, structural, and microstructural changes in presymptomatic C9orf72 carriers younger than 40 years. JAMA Neurol. 2018;75:236-245.

38. Bocchetta M, Todd EG, Peakman G, et al. Differential early subcortical involvement in genetic FTD within the GENFI cohort. Neuroimage Clin. 2021;30:102646.

39. Cudkowicz ME, McKenna-Yasek D, Chen C, Hedley-Whyte ET, Brown RH. Limited corticospinal tract involvement in amyotrophic lateral sclerosis subjects with the $\mathrm{A} 4 \mathrm{~V}$ mutation in the copper/zinc superoxide dismutase gene. Ann Neurol. 1998;43(6) 703-710.

40. Mahoney CJ, Downey LE, Ridgway GR, et al. Longitudinal neuroimaging and neuropsychological profiles of frontotemporal dementia with C9ORF72 expansions. Alzheimers Res Ther. 2012;4(5):41.

41. Irish M, Devenney E, Wong S, et al. Neural substrates of episodic memory dysfunction in behavioural variant frontotemporal dementia with and without C9ORF72 expansions. Neuroimage Clin. 2013;2:836-843.

42. Lee SE, Sias AC, Mandelli ML, et al. Network degeneration and dysfunction in presymptomatic C9ORF72 expansion carriers. Neuroimage Clin. 2016;14:286-297.

43. De Vocht J, Blommaert J, Devrome M, et al. Use of multimodal imaging and clinical biomarkers in presymptomatic carriers of C9orf72 repeat expansion. JAMA Neurol. 2020;77(8):1008-1017

44. Wolff M, Vann SD. The cognitive thalamus as a gateway to mental representations. J Neurosci. 2019;39(1):3-14

45. Stoodley CJ, Schmahmann JD. Evidence for topographic organization in the cere bellum of motor control versus cognitive and affective processing. Cortex. 2010;46(7) 831-844.

46. Montembeault M, Sayah S, Rinaldi D, et al. Cognitive inhibition impairments in presymptomatic C9orf72 carriers. J Neurol Neurosurg Psychiatry. 2020;91(4):366-372. 


\section{Neurology}

\section{Structural MRI Signatures in Genetic Presentations of the Frontotemporal Dementia/Motor Neuron Disease Spectrum \\ Edoardo Gioele Spinelli, Alma Ghirelli, Silvia Basaia, et al.}

Neurology 2021;97;e1594-e1607 Published Online before print September 20, 2021

DOI 10.1212/WNL.0000000000012702

This information is current as of September 20, 2021

\section{Updated Information \& Services}

References

Citations

Subspecialty Collections

Permissions \& Licensing

Reprints including high resolution figures, can be found at: http://n.neurology.org/content/97/16/e1594.full

This article cites 46 articles, 7 of which you can access for free at: http://n.neurology.org/content/97/16/e1594.full\#ref-list-1

This article has been cited by 2 HighWire-hosted articles: http://n.neurology.org/content/97/16/e1594.full\#\#otherarticles

This article, along with others on similar topics, appears in the following collection(s):

\section{All Genetics}

http://n.neurology.org/cgi/collection/all_genetics

Amyotrophic lateral sclerosis

http://n.neurology.org/cgi/collection/amyotrophic_lateral_sclerosis_ Frontotemporal dementia

http://n.neurology.org/cgi/collection/frontotemporal_dementia MRI

http://n.neurology.org/cgi/collection/mri

Volumetric MRI

http://n.neurology.org/cgi/collection/volumetric_mri

Information about reproducing this article in parts (figures,tables) or in its entirety can be found online at:

http://www.neurology.org/about/about_the_journal\#permissions

Information about ordering reprints can be found online:

http://n.neurology.org/subscribers/advertise

Neurology ${ }^{\circledR}$ is the official journal of the American Academy of Neurology. Published continuously since 1951, it is now a weekly with 48 issues per year. Copyright Copyright ( 2021 The Author(s). Published by Wolters Kluwer Health, Inc. on behalf of the American Academy of Neurology.. All rights reserved. Print ISSN: 0028-3878. Online ISSN: 1526-632X.

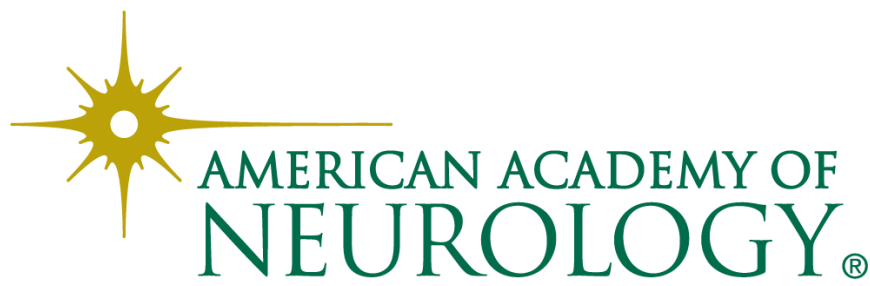

